\title{
Variable de género y competencia colocacional léxica en el aula de ELE
}

\author{
Virginia de Alba QuiÑones \\ Universidad Pablo de Olavide
}

Recibido: 14 noviembre 2014 / Aceptado: 1 febrero 2015

ISSN: $1697-7467$

\begin{abstract}
RESUMEN: En este trabajo se pretende analizar desde la perspectiva de la diferencia de género la competencia colocacional que presentan 99 alumnos de español como lengua extranjera de la Freie Universität Berlin. Se elidió el verbo de 10 enunciados donde se ofrecía de manera contextualizada la colocación y se les pidió que sin ayuda rellenaran el hueco (la traducción de la colocación al alemán estaba reflejada al final de cada frase). El resultado de este trabajo parece desestimar que la variable del género incida en el conocimiento de estas unidades plurimembres.

Asimismo, hacemos unas aportaciones didácticas para desarrollar adecuadamente la competencia colocacional.

Palabras clave: Español como lengua extranjera (ELE), competencia colocacional, género y L2, didáctica del léxico en ELE.
\end{abstract}

\section{Gender Variability and Lexical Collocational Competence in Spanish as a Foreing Language}

\begin{abstract}
This paper aims to analyze the collocational competence of 99 Spanish as a foreign language students at Freie Universität Berlin from a gender perspective. They were asked to complete 10 phrases featuring verb-noun collocation from which the verb had been removed (a German equivalent translation was provided at the end of each sentence). The result of this study seems to dismiss the idea of a gender variable regarding knowledge of lexical collocations. Additionally, we offer some teaching ideas for adequately developing collocational competence.

Key words: Spanish as a Foreign Language, collocational competence, gender and L2, Teaching Vocabulary in Spanish as Foreign Language.
\end{abstract}

\section{InTRODUCCIÓN}

Asumir el complejo concepto de competencia comunicativa en el ámbito de la enseñanza de lenguas extranjeras implicó un completo cambio en la concepción del proceso de enseñanza y aprendizaje y de lo que entendemos por lengua.

Tradicionalmente, la enseñanza de una lengua extranjera se materializaba en la enseñanza deductiva de la gramática y el aprendizaje del vocabulario mediante glosarios descontextualizados. En la actualidad, nuestras aulas de enseñanza de L2 se convierten en verdaderos crisoles metodológicos, donde podemos encontrar infinidad de técnicas basadas todas en los mismos principios: participación activa del aprendiz tanto en el proceso de enseñanza, 
como en el de aprendizaje; cambio de actitud frente al error; ofrecer la mayor cantidad de input posible; importancia de la oralidad; estimulación de las destrezas productivas; objetivo centrado en la comunicación auténtica... Muy ampliamente conocida es la idea de que cuando las personas viajan a un país extranjero no se llevan gramáticas, sino diccionarios (Krashen, 1987) porque son las palabras las que, en realidad, nos permiten comunicarnos. Para llegar a esta conclusión, uno de los factores determinantes ha sido la constatación de que los problemas relacionados con la competencia léxica no solo dificultan la comunicación, sino que pueden llegar a impedirla totalmente, mientras que un error gramatical es fácilmente subsanable, en la mayoría de los casos, por parte de un nativo (Lewis, 2000).

Por este motivo, la competencia léxica centra nuestro trabajo y dentro de las unidades que la integran, un subgrupo dentro de las estructuras plurimembres, las colocaciones.

\section{Aprender una unidad léxica}

El objetivo de la competencia léxica es el conocimiento de las unidades léxicas, entendidas como 'las piezas básicas de aprendizaje de vocabulario que se identifican con una unidad conceptual'. Estas se diferencian de la palabra (que se define gracias a criterios formales como 'el segmento de la cadena hablada que se refleja en la escritura como una secuencia de letras separada por espacios en blanco o signos de puntuación y que posee, al menos, un significado') al encontrarse en un nivel superior: una unidad léxica (UL) puede estar formada por una o por varias palabras.

Somos conscientes tanto de que el complejo proceso cognitivo que supone aprender, conocer, reconocer y usar adecuadamente una unidad léxica difícilmente alcanza todos los subaprendizajes que se señalan (Nation [2001] o Izquierdo [2004]), pero uno de ellos, el dominio de las estructuras pluriverbales, más concretamente el dominio de las colocaciones léxicas —entendidas como unidades del discurso repetido (Coseriu, 1977) o como chunks (Lewis, 2000) - serán el objetivo de la investigación que presentamos.

Hill, seguidor del enfoque léxico, señala que enseñar explícitamente las colocaciones está respaldado porque existen unas reglas que subyacen bajo las combinaciones de palabras; además, según afirma (Hill 2000: 53): "Estimates vary, but it is possible that up to $70 \%$ of everything we say, hear, read or write is to be found in some form of fixed expression". Entender que las palabras funcionan en bloque, facilita, entre otras cuestiones, fluidez al discurso del aprendiz, comprender y producir discursos complejos y la correcta pronunciación de los elementos léxicos.

\section{Las colocaciones. Características}

Mucho se ha discutido sobre si las colocaciones forman parte o no de la Fraseología y sobre sus características. Lo cierto es que los primeros estudios sobre estas unidades se dieron en el contextualismo inglés y parece haber unanimidad en señalar a Firth (1957) como el primer investigador en usar el término collocation. No obstante, compartimos con Benson (1985), Corpas Pastor (1996 y 2001), Koike (2001), Castillo Carballo (1998) o Alonso Ramos 
(1994-95 y 2012), entre otros, que estas unidades trascienden al hecho de la coaparición en el discurso y que se establece entre sus integrantes una relación léxico-semántica; por tanto, en este trabajo se asume que las colocaciones son unidades que pertenecen al campo de investigación de la Fraseología y que cuentan con las siguientes características (seguimos a Koike, 2001):

El criterio formal: las colocaciones están formadas por dos o más palabras que coaparecen frecuentemente; esta atracción está determinada por el uso y sus componentes pueden cambiarse por otros sin que por ello se pierda el sentido.

El criterio semántico: entre esta combinación de palabras se establece un vínculo jerarquizado que condiciona una relación típica y precisa, que participa del concepto de transparencia y de cohesión semántica.

Todos los rasgos anteriormente señalados son los que caracterizan a las unidades que en la Fraseología se conocen por colocaciones. Sin embargo, desde la praxis del aula de ELE, compartimos su división de estas estructuras en prototípicas y no prototípicas. Ya que como señala Higueras García (2006:26): "Muchos de los ejemplos que se emplean en actividades no serían consideradas como tales por la mayoría de los lingüistas". De esta manera, las colocaciones no prototípicas estarían en un punto del contínuum establecido entre las combinaciones libres de palabras y lo que tradicionalmente se ha venido conociendo como colocaciones desde el ámbito de la Fraseología, ya que no participan en el mismo grado de la restricción léxica, de la tipicidad o de la direccionalidad.

\section{ObJetivo de la investigación}

Desde los primeros acercamientos de finales del siglo pasado -Pavlenko (1999, 2001, 2004) o Singleton (1999), por ejemplo— hasta los últimos estudios —Jiménez Catalán (2003), Corpas Arellano (2010), Pear (2011) y Pear y Barret (2013), entre otros-, la línea de investigación sobre la variable sexo o género (en este trabajo se usan de manera indistinta los dos términos) se ha centrado en indagar si la población masculina y la femenina desarrollaban las mismas o diferentes tipos de estrategias de aprendizaje y en qué medida. Las conclusiones de diferentes estudios ponen de manifiesto que no son las mismas estrategias las que emplean los varones y las mujeres y, además, que estas últimas hacen uso de un repertorio más variado, aunque hay estudios, como el de Wharton (2000), que señalan justo lo contrario.

No obstante, en una encuesta realizada a profesores de L2 de todos los niveles educativos, estos señalan que (Rodríguez-Pérez 2014:195): "Los informantes aclaran que las diferencias de género se deben a motivos socio-culturales (especialmente la influencia del profesor y de la familia) y de madurez del alumno, no a causas de origen innato." En esta línea, San Mateo Valdehíta (2012) llegó a la conclusión que las variables individuales y sociales de los estudiantes no inciden significativamente en el aprendizaje de vocabulario en la L2, sino que son las actividades empleadas en dicho aprendizaje las que determinan el éxito del proceso.

Así, aunque somos conscientes de que existen más variables individuales que inciden en el éxito del acercamiento de un aprendiz a la L2 (motivación, expectativas, autoestima, confianza...), el objetivo del presente estudio es determinar si el factor sexo incide en el dominio colocacional que presentan los aprendices de español. 


\section{LA INVESTIGACIÓN}

\subsection{Participantes}

Un total de 103 estudiantes de 5 niveles diferentes de ELE del Sprachenzentrum de la Universidad Libre de Berlín tomaron parte en la investigación ${ }^{1}$, sin embargo descartamos analizar las pruebas realizadas por 4 de ellos, ya que uno era un estudiante español con una beca Erasmus y 3 manifestaron su condición de bilingües. Por lo tanto, el presente estudio está basado en un corpus obtenido a partir de 99 informantes (de ellos, 32 eran de género masculino y 67 eran féminas), su distribución queda reflejada a continuación:

Tabla 1. Distribución de los estudiantes

\begin{tabular}{|c|c|c|}
\hline \multirow[t]{2}{*}{ NIVEL } & \multicolumn{2}{|c|}{ NÚMERO DE ESTUDIANTES } \\
\hline & Totales & Por género \\
\hline \multirow[t]{2}{*}{ B.1.1 } & \multirow[t]{2}{*}{25} & 100 \\
\hline & & 15 운 \\
\hline \multirow[t]{2}{*}{ B.2.1 } & \multirow[t]{2}{*}{21} & 70 \\
\hline & & 15 우 \\
\hline \multirow[t]{2}{*}{ B.2.2 } & \multirow[t]{2}{*}{35} & 110 \\
\hline & & 24 운 \\
\hline \multirow[t]{2}{*}{ C.1.1 } & \multirow[t]{2}{*}{13} & $3 \delta$ \\
\hline & & $10 ㅇ$ \\
\hline \multirow[t]{2}{*}{ C. 1.2} & \multirow[t]{2}{*}{4} & $1 \delta$ \\
\hline & & 3 우 \\
\hline
\end{tabular}

\subsection{Procedimiento}

Existen básicamente tres formas diferentes de estudiar el uso y el conocimiento productivo de las colocaciones:

- Realizar un estudio de análisis de corpus

- Analizar creaciones textuales (libres, semidirigidas o dirigidas)

- Recurrir a ejercicios donde falte uno de los colocados (con o sin una orientación contextualizada de las estructuras)

Para la realización del presente trabajo, nos decantamos por la última opción, concretamente optamos por usar diez enunciados escritos en español en los que se omitiría uno de los dos elementos que constituyen la colocación y añadir al final la traducción a L1 del grupo de estudiantes que iba a tomar parte en la investigación, el alemán en este caso. Tras determinar el formato de la investigación, la primera cuestión que nos planteamos fue la búsqueda de un repertorio donde existieran colocaciones acordes con todos los niveles de dominio lingüístico. Para ello, se partió de varias premisas:

- De las distintas taxonomías que se ofrecen en lengua española para estas estructuras plurimembres, nos hemos decantado por las formadas por sustantivo

${ }^{1}$ Quiero manifestar mi enorme agradecimiento por la colaboración prestada por el profesorado del área de

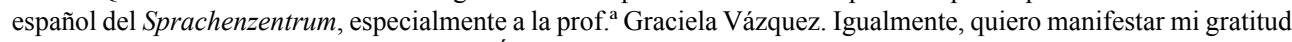
por la colaboración desinteresada del Prof. Ángel Tenorio en la realización del análisis estadístico. 
(en función de objeto) + verbo por ser las de mayor frecuencia de aparición en el discurso. Dado el carácter direccional de la estructura, fue el colocativo el que se determinó que se elidiría.

- Como el grupo meta estaba constituido por aprendices de la Universidad Libre de Berlín, se trató de evitar que los colocativos en español fueran exactamente una traducción en la colocación en alemán, ya que como señalan Nesselhauf (2003) o Webb y Kagimoto (2011:260): "the role of the L1 was far greater than initially expected with it being found to have an overwhelming influence on the amount and type of collocation error produced". Queremos detenernos aquí para señalar una cuestión que nos parece pertinente: en este mundo globalizado lo usual es encontrar hablantes multilingües, con su consiguiente lexicón multilingüe (Wei, 2003). Por este motivo, creemos que es una entelequia tratar en una investigación a un colectivo como homogéneo lingüísticamente, especialmente, en una urbe tan multicultural como es Berlín, ya que algunos de los estudiantes que han tomado parte en este trabajo (o sus familias) provienen de otro/s país/es, y para los estudiantes de origen alemán, el español es, al menos, la segunda L2 que estudian. Por todo lo anterior, lo que asumimos para el diseño de este estudio es que los estudiantes poseen un nivel lo suficientemente alto en alemán como para justificar su presencia en un centro de educación universitario y público en Alemania.

- Tanto en la confección de la colocación en alemán, como en español, se trató de que el verbo que constituye la colocación no fuera un verbo puente, sino uno con significado pleno.

Tras asumir estas premisas, nos centramos en la búsqueda del repertorio colocacional que nos serviría de referente para la realización de la investigación y recurrimos al Plan curricular del Instituto Cervantes. Niveles de referencia para el español (Instituto Cervantes, 2006) (PCIC en adelante), que es la concreción del Marco Común de Referencia Europeo para las Lenguas (Ministerio de Educación, Cultura y Deporte, 2002) (MCER en lengua española), lo que era especialmente necesario en su vertiente de ELE/L2.

Se desarrollan en el PCIC los siguientes componentes de la competencia comunicativa: el gramatical, el pragmático-discursivo, el nocional, el cultural y el de aprendizaje. Siguiendo las propias palabras del PCIC (2006), se definen "los contenidos de carácter léxico-semántico desde una perspectiva de análisis de carácter nocional, con el desarrollo de dos inventarios de vocabulario en términos de nociones generales y específicas.". El vocabulario aparece estructurado según los 6 niveles de dominio lingüístico del MCER y agrupado en conjuntos semántico-gramaticales, a modo de conceptos que aglutinan tanto lexías simples como complejas y otros tipos de estructuras. Para la búsqueda de las unidades plurimembres que iban a formar parte de este estudio, nos centramos en el apartado de "nociones generales" dada la naturaleza que este presenta, ya que al no estar vinculado a un ámbito específico, los aprendices deberían dominar estas estructuras con independencia del contexto comunicativo. Sin embargo, no fue posible extraer todos los ejemplos que necesitábamos de allí, ya que no aparecían suficientes colocaciones para todos los niveles lingüísticos que presentaban los aprendices, así que se tuvo que recurrir al inventario de las "nociones específicas". 
Cuando se contactó con la plantilla docente del área de Español del Centro de Idiomas de la Universidad Libre de Berlín, se nos informó de que estos serían los niveles en los que podríamos pasar la prueba: B.1.1, B.2.1, B.2.2, C.1.1 y C.1.2; por tanto, teníamos grupos que participan del enfoque ramificado del MCER. Para solventar esta situación, se procedió a tomar 5 de las colocaciones de las que están recogidas en ese nivel y 5 del inmediatamente superior. Finalmente, diseñamos enunciados en los que insertamos las colocaciones, como puede verse en los siguientes ejemplos de los niveles B.2.1 y C.2.1 respectivamente:

- Todo estaba mal en el hotel: la habitación tenía mucho ruido, el cuarto de baño estaba sucio y el personal era muy poco amable; así que una reclamación (eine Reklamation schreiben).

- En España es muy importante el pésame cuando muere alguien (das Beileid aussprechen).

\subsection{Tiempo de realización de la prueba}

Durante la semana del 12 al 16 de mayo de 2014 se procedió a la recopilación del corpus y cada alumno tuvo 10 minutos para rellenar, sin ningún tipo de apoyo, el colocativo, que estaba omitido en cada una de los 10 enunciados.

\section{Los DATOS}

Tras la recopilación del corpus, se procedió a su análisis y a la primigenia clasificación de elección correcta, incorrecta o ausencia de contestación, hubo que incluir el apartado "Registro no adecuado", ya que hubo contestaciones correctas, pero por debajo del registro que se esperaba que tuvieran los estudiantes, por ejemplo: uso de la colocación dar valor en lugar de insuflar valor o abrir un congreso en lugar de inaugurar un congreso.

En las siguientes tablas, recogemos los datos obtenidos según los niveles que presentaban los estudiantes:

Tabla 2. Datos por nivel lingüístico

\begin{tabular}{|l|l|l|l|l|l|}
\hline & NIVEL B.1.1 & NIVEL B.2.1 & NIVEL B.2.2 & NIVEL C.1.1 & NIVEL C.1.2 \\
\hline CORRECTAS & 70 & 54 & 98 & 64 & 27 \\
\hline INCORRECTAS & 73 & 101 & 156 & 34 & 10 \\
\hline REGISTRO NO ADECUADO & 18 & 6 & 5 & 23 & 3 \\
\hline VACÍOS & 89 & 49 & 91 & 9 & 0 \\
\hline
\end{tabular}


Reflejamos los resultados de la tabla anterior, haciendo una división por el género de los aprendices:

Tabla 3. Datos por género y por nivel lingüístico

\begin{tabular}{|l|l|l|l|l|l|l|l|l|l|l|}
\hline & \multicolumn{2}{l|}{$\begin{array}{l}\text { NIVEL } \\
\text { B.1.1.1 }\end{array}$} & \multicolumn{2}{l|}{$\begin{array}{l}\text { NIVEL } \\
\text { B.2.1 }\end{array}$} & \multicolumn{2}{l|}{ NIVEL B.2.2 } & \multicolumn{2}{l|}{$\begin{array}{l}\text { NIVEL } \\
\text { C.1.1 }\end{array}$} & \multicolumn{2}{l|}{$\begin{array}{l}\text { NIVEL } \\
\text { C.1.2 }\end{array}$} \\
\hline & 0 & + & 0 & $q$ & 0 & $q$ & 0 & + & 0 & + \\
\hline CORRECTAS & 31 & 39 & 13 & 41 & 30 & 68 & 13 & 51 & 6 & 21 \\
\hline INCORRECTAS & 29 & 44 & 39 & 62 & 48 & 108 & 8 & 26 & 4 & 6 \\
\hline $\begin{array}{l}\text { REGISTRO NO } \\
\text { ADECUADO }\end{array}$ & 7 & 11 & 0 & 6 & 2 & 3 & 4 & 19 & 0 & 3 \\
\hline VACÍOS & 33 & 56 & 18 & 31 & 30 & 61 & 5 & 4 & 0 & 0 \\
\hline
\end{tabular}

\section{Análisis de los datos}

\section{1. Análisis descriptivo}

Si analizamos los porcentajes de los grupos en cada uno de los apartados de la taxonomía, podemos constatar lo siguiente:

\subsubsection{Respuestas correctas}

El mayor porcentaje de colocaciones correctas lo presenta el nivel C.1.2 con el $67,5 \%$ de aciertos, en el extremo contrario encontramos el grupo B.2.1 con el $25 \%$ de respuestas correctas. El alumnado de los grupos C.1.1 y C.1.2 es el que presenta mayor nivel de competencia colocacional, con un $49,2 \%$ y un $67,5 \%$ respectivamente.

Estos resultados vienen a apoyar el estudio de Laufer y Waldman (2011) donde señalan que solo en los niveles avanzados aumentan significativamente las colocaciones correctas.

\subsubsection{Respuestas incorrectas}

El mayor porcentaje de colocaciones incorrectas lo presenta el grupo B.2.1, con el 48\%, mientras que el menor lo encontramos el nivel C.1.2 (25\%). Puede resultar llamativo que el B.1.1. posea un nivel inferior de colocaciones incorrectas que el B.2.1, sin embargo, no debemos obviar que el porcentaje de respuestas sin contestar es mayor en el B.1.1.

\subsubsection{Registro no adecuado}

Este nivel de clasificación hace referencia a la variedad diafásica y lo que nos sirve de referencia es el enriquecimiento en el registro de los aprendices de ELE.

En el nivel C.1.1. encontramos el mayor índice de registros no adecuados (el 17\%) y este se manifiesta especialmente en el uso de la colocación sentir sensaciones (que se correspondería con las colocaciones implícitas definidas por Travalia [2006]) en lugar de experimentar sensaciones. 


\subsubsection{Respuestas vacías}

El grupo con mayor índice de enunciados sin respuesta presenta es el de menor nivel lingüístico, el B.1.1, con el 35\% y en el extremo contrario, aparece el del nivel C.1.2, donde no encontramos ninguna oración sin contestar.

\subsection{Análisis estadístico}

\subsubsection{Explicación del estudio y los datos tratados}

El análisis de la varianza (ANOVA en adelante) necesita que las variables que se van a estudiar mediante el factor explicativo sean aleatorias (se puede presuponer en el caso en cuestión); independientes (lo presuponemos porque a priori no tienen por qué tener relación), que se ajusten al criterio de normalidad (sin tener en cuenta el factor); y homogeneidad de las varianzas (que la dispersión de la muestra sea similar para el factor explicativo).

Es pertinente distinguir si las muestras presentan más de 30 individuos o una cifra inferior a esta cantidad. En el primer caso, el test de normalidad es el de Kolmogorov-Smirnov y en el segundo el de Shapiro-Wilk. En cualquiera de los dos test, la normalidad se supone como cierta si se obtiene una significatividad superior al 0.05 .

Estudiada la normalidad, a las variables que siguen una distribución normal se les ha aplicado una prueba $t$ de Student para estudiar si el comportamiento de dicha variable depende del factor que se quiere considerar (en este caso el factor género). Si la significatividad resulta superior a 0.05 , entonces se asume que no hay diferencias significativas en la variable estudiada en relación al factor género. Para el caso de las variables de estudio que no seguían una variable normal, existen dos opciones (al menos las más habituales) para estudiar la influencia del factor género: unA es la U de Mann-Whitney y el otra es la $\mathrm{W}$ de Wilcoxson. Ambos son equivalentes a la hora de asumir o desechar la hipótesis nula de igualdad de comportamiento de la variable respecto del factor de estudio; se ha realizado la U por ser más conocida,

\subsubsection{Análisis estadísticos por grupo}

- Nivel B.1.1

La normalidad se comprueba con la prueba de Shapiro-Wilk una vez realizado el análisis, comprobamos el p-valor de las cuatro variables consideradas que son $0.391,0.263$ y 0.201 para las variables "Correcto", "Incorrecto" y "Vacío", respectivamente. Esto nos permite afirmar que las tres variables siguen una distribución normal. Sin embargo, la variable "No adecuado" tiene p-valor 0.000 ; así debemos considerar que no sigue una distribución normal, como pone de manifiesto la siguiente tabla (únicamente reflejamos este tipo de tablas relacionadas con el análisis estadístico en este nivel lingüístico): 
Tabla 4. Análisis de la normalidad

Pruebas de normalidad

\begin{tabular}{|l|l|l|l|l|l|l|}
\hline & \multicolumn{4}{l}{ Kolmogorov-Smirnov ${ }^{\mathrm{a}}$} & \multicolumn{2}{l|}{ Shapiro-Wilk } \\
\cline { 2 - 7 } & Estadístico & $\mathrm{gl}$ & $\mathrm{Sig}$ & Estadístico & $\mathrm{gl}$ & Sig. \\
\hline Correcto &, 140 & 25 &, $200^{*}$ &, 959 & 25 &, 391 \\
Incorrecto &, 161 & 25 &, 094 &, 951 & 25 &, 263 \\
No adecuado &, 377 & 25 &, 000 &, 709 & 25 &, 000 \\
Vacío &, 144 & 25 &, 195 &, 946 & 25 &, 201 \\
\hline
\end{tabular}

*. Este es un límite inferior de la significación verdadera.

a. Corrección de la significación de Lilliefors

En las variables que siguen distribución normal se ha realizado una comparación de las medias mediante la prueba t de Student, que es $0.443,0.962$ y 0.621 , respectivamente. Así, se supone que las tres categorías estudiadas no presentan diferencias significativas en su comportamiento en relación al factor género.

Como realmente el factor "sexo" solo tiene dos valores, bastaría estudiar la prueba t de Student para comprobar su influencia en las categorías. No obstante, también se ha realizado un ANOVA de un factor mediante un contraste polinómico de tipo cúbico (el más habitual) con una comparación post-hoc de tipo Tukey, ya que hemos podido asumir que las varianzas son iguales (por el test de Levene ya realizado). Miramos el p-valor de cada una de las categorías y observamos que todas lo presentan superior a 0.05 y hemos de presuponer que el factor "sexo" no influye en ninguna de las categorías consideradas.

Para la categoría "No adecuado", al no seguir una distribución normal, hemos de emplear el test U de Mann-Whitney como prueba no paramétrica, el p-valor resulta ser de 0.947, podemos afirmar, igualmente, que el factor "sexo" no influye en la categoría "No adecuado".

Tabla 5. Análisis de la categoría "No adecuado"

Estadísticos de contraste $^{a}$

\begin{tabular}{|l|l|}
\hline & NoAdecuado \\
\hline U de Mann-Whitney & 74,000 \\
W de Wilcoxon & 129,000 \\
Z &,- 066 \\
Sig. asintót. (bilateral) &, 947 \\
Sig. exacta [2*(Sig. &, $978^{\mathrm{b}}$ \\
unilateral)] & \\
\hline
\end{tabular}

a. Variable de agrupación: Sexo

b. No corregidos para los empates. 
- Nivel B.2.1

La normalidad se comprueba nuevamente con la prueba de Shapiro-Wilk y lo p-valores son 0.137 y 0.223 para las variables "Correcto" e "Incorrecto", respectivamente. Así, estas dos variables siguen una distribución normal. Por su parte, las variables "No adecuado" y "Vacío" tienen p-valor 0.000 y 0.033 , por lo que debemos asumir que no siguen una distribución normal.

Estudiamos el comportamiento del factor "sexo" para las variables que siguen distribución normal mediante la prueba $\mathrm{t}$ de Student y se observa que el p-valor del test de Levene es superior a 0.05 por lo que en los dos casos tenemos homogeneidad de las varianzas y el resultado de la prueba t de Student es 0.156 y 0.121 , respectivamente; de lo que se deduce que las dos categorías estudiadas no presentan diferencias significativas en su comportamiento en relación al factor género.

Para las categorías "No adecuado" y "Vacío", al no seguir una distribución normal, hemos de emplear el test U de Mann-Whitney como se indicó antes. En el caso de la variable "Vacío" se obtiene un p-valor de 0.858 que nos hace asumir la no influencia del factor "sexo" en su comportamiento. En el caso de la variable "No adecuado", el p-valor es de 0.055, que está en la frontera del valor que nos permite asumir la hipótesis nula, por la que el comportamiento de la variable no viene afectado por el valor "Sexo", pero dicha asunción es límite.

- Nivel B.2.2

Nuevamente se estudia si las variables analizadas siguen una distribución normal para la población completa, para ello usamos la prueba de Kolmogorov-Smirnov. En las cuatro variables consideradas, los p-valores obtenidos son todos inferiores a 0.05 por lo que hemos de asumir que ninguna de ellas sigue una distribución normal. Debido a este hecho, solo es factible el análisis de la influencia del factor "sexo" mediante la prueba U de MannWhitney. El p-valor de las categorías estudiadas es, respectivamente, 0.676, 0.918, 0.498 y 0.746 . Por tanto, en ninguna de las variables podemos asumir que el factor "sexo" tiene influencia en dichas categorías.

- Nivel C.1.1

En este caso, la normalidad se comprueba con la prueba de Shapiro-Wilk. De las cuatro variables consideradas, solo el p-valor 0.110 es superior a 0.05 para la variable "No adecuado", que podemos asumir sigue una distribución normal. Sin embargo, los p-valores de las otras tres variables son inferiores a $0.05 \mathrm{y}$, por tanto, ninguna sigue una distribución normal. Como consecuencia, solo es factible el análisis de la influencia del factor "sexo" mediante la prueba U de Mann-Whitney. El p-valor de las categorías estudiadas es, respectivamente, 0.472, 0.856 y 0.040 . Por tanto, en las variables "Correcto" e "Incorrecto" no se puede asumir la influencia del factor "sexo" en el comportamiento de las variables. En el caso de la variable "Vacío", el p-valor conlleva que podamos asumir la influencia del factor "sexo" en el comportamiento de la variable y es que, especialmente en este nivel, se observa una tendencia generalizada en todas las muestras: que las féminas no suelen dejar preguntas sin contestar, mientras que los varones en nuestro corpus siempre dejan alguna pregunta sin responder.

Como la variable "No adecuado" sigue una distribución normal, estudiamos el comportamiento del factor "sexo" mediante la prueba t de Student. Como el p-valor del test de Levene es superior a 0.05 , tenemos homogeneidad de las varianzas y el resultado de la prueba t de Student es 0.376, no influyendo el factor "sexo" en el comportamiento de la variable. 


\section{- Nivel C.1.2}

La normalidad se comprueba con la prueba de Shapiro-Wilk. De las cuatro variables consideradas, la variable "Vacío" toma valor constante y, por tanto, no procede compararla con una distribución normal ni realizar un estudio en función del factor "sexo".

En las otras tres variables, el p-valor es superior a 0.05 y podemos asumir que siguen una distribución normal.

Por todo lo anterior, podemos aplicar tanto una prueba t de Student como un ANOVA para determinar la influencia del factor "sexo" para las tres variables de estudio. Debido al tamaño muestral, no se puede hacer el test de homogeneidad de la varianza. En cualquier caso, el p-valor es superior a 0.05 en las tres variables para el test $\mathrm{t} y$, por tanto, el factor "sexo" no influye en el comportamiento de dichas variables.

\section{Aportaciones didácticas a la enseñanza de las colocaciones}

Desde el inicio de los estudios en torno a la competencia colocacional se tomó conciencia de que esta era un área especialmente complicada para los aprendices de lenguas extranjeras. No obstante, es igualmente cierto que existe lo que podríamos denominar "universales fraseológicos"; por ello, hay veces en las que el aprendiz puede deducir el significado de distintas estructuras plurimembres apoyándose en el conocimiento que tenga de otra/s lengua/s (de Alba, 2014). Así, distintos estudios (Altenberg y Granger, 2001 o Nesselhauf, 2003) han demostrado cómo el universo colocacional es difícil de aprehender para los estudiantes de L2; aunque existen otros trabajos (de Alba 2014) donde se constata que los aprendices tienen tendencia a usar estrategias de traducción de los colocados. No obstante, queremos destacar que cuando no existe una estructura formada por unidades análogas, las colocaciones frecuentemente resultan imposibles de producir para el estudiante.

Las colocaciones están presentes en cualquier discurso y, como señala Handl (2008:47): "For everyday conversation and standard writing tasks, however, collocation is much more important, since the incorrect use of a word in the context immediately unmasks the nonnative speaker". Este desenmascaramiento, dada la usual transparencia semántica de estas estructuras plurimembres, se concentra en la producción, no en la recepción de los mensajes en L2, por lo que para desarrollar la competencia colocacional, debemos estimular las producciones de nuestros estudiantes en la L2.

En nuestra tarea docente es importante no solo aumentar cuantitativamente el vocabulario de nuestros estudiantes, sino hacerlo cualitativamente, como señaló acertadamente Woolard (2000:31): "learning more vocabulary is not just learning new words, it is often learning familiar words in new combinations". Para introducir con la mayor eficacia una UL en el lexicón, (o extender la red de un elemento que ya existiera) no debemos obviar que es esencial esta triada, exposición-cognición-producción:

a) Exponer al aprendiz a la máxima cantidad de input contextualizado que nos sea posible, siempre en circunstancias comunicativas lo más cercanas a la realidad y a los intereses de los estudiantes.

b) No debemos obviar que el aprendizaje se produce tanto de manera incidental como intencional. Si centramos el foco de interés sobre las colocaciones, nos 
decantamos por poner de relieve aquellas unidades plurimembres que no se construyen de igual modo en la L1 y la L2 de los estudiantes: por tanto, nos inclinamos por la enseñanza explícita de estas estructuras y por el aprendizaje implícito de aquellas que sean el resultado de la traducción de los colocados entre la L1 y la L2.

c) Tras esto, debemos desarrollar un entorno comunicativo en el aula y estimular la creación de entornos fuera de ella, ya que debemos asimilar que, en el reducido tiempo de las clases, no podemos proporcionar a nuestros estudiantes ni el input necesario ni las circunstancias para que el output se produzca con la frecuencia deseada para conseguir asentar el conocimiento y el uso del vocabulario.

En relación con la cuestión de cuándo debe producirse el acercamiento al universo colocacional, defendemos junto con Fernández Lázaro (2014) que se deben enseñar estas estructuras desde los niveles iniciales y la elección de las unidades debe venir determinada por la frecuencia en el discurso de las unidades y por la adecuación a su nivel. Queremos incidir en los resultados del estudio llevado a cabo por Ellis et al. (2008) en el que se demostró que aprendices principiantes eran capaces de reconocer estructuras plurimembres si estas aparecían frecuentemente en el input.

\section{Conclusiones}

Es compartido por todos que tanto el proceso de enseñanza como el de aprendizaje son altamente complejos y que son muchos los factores que inciden en ambos. A pesar de que es un hecho constatado que la subcompetencia léxica ha sido infravalorada en el pasado, sigue notándose la ausencia de estudios que indaguen sobre la adquisición y el uso del vocabulario de los aprendices de L2. Esta escasez todavía se hace más patente si al vocabulario unimos la variable género. Por ello, en este estudio nos hemos centrado en el análisis de la variable género y la competencia colocacional. Tradicionalmente, se ha venido asumiendo que las diferencias de género podían materializarse en un mayor éxito de las féminas frente a los varones en la adquisición de L2. Sin embargo, los resultados de nuestra investigación demuestran que la variable género no parece tener incidencia en los resultados del test de competencia colocacional de los estudiantes que han participado en el estudio. Creemos, por tanto, que deben desarrollarse más estudios en esta línea.

Respecto de las implicaciones didácticas sobre la enseñanza de las colocaciones, queremos destacar que apoyamos la tesis de abordar su enseñanza desde los inicios del proceso de aprendizaje; que debemos estimular el input y las posibilidades de output de estas unidades al máximo, tanto dentro como fuera del aula de ELE; y que, siempre que sea posible, se podría facilitar la enseñanza de estas unidades pluriverbales haciendo un abordaje desde la lingüística contrastiva, para realizar un aprendizaje explícito centrado en aquellas estructuras cuyos colocados difieran entre la L1 de los aprendices y el español. 


\section{ReFERENCIAS}

Alba Quiñones, V. de (2011). "La competencia léxica. Una propuesta de actividades sobre los campos léxicos para las clases de ELE", en MarcoELE, 13: 1-14, disponible en: http:// marcoele.com/la-competencia-lexica/, consultado el 22 de septiembre de 2014.

Alba Quiñones, V. de (2014). "El uso de las colocaciones en muestras escritas de aprendices de español. Análisis e implicaciones metodológicas", en Estudios de Lingüística de la Universidad de Alicante, 28: 151-176.

Alonso Ramos, M. (1994-1995). "Hacia una definición del concepto de colocación: de J. R. Firth a I. Mel'cuk", en Revista de Lexicografía, 1: 9-28.

Alonso Ramos, M. (2012). "Sobre por qué un grupo de palabras puede ser una sola palabra sin ser paradójico", en E. Tomás Moro del Arco (ed.) Neología y creatividad lingüística. Valencia: Universidad de Valencia, 115-136.

Altenberg, B. y Granger, S. (2001). "The Grammatical and Lexical Patterning of MAKE in Native and Non-Native Student Writing", en Applied Linguistics, 22, 2: 173-195.

Benson, M. (1985). "Collocations and idioms", en R. Ilson (ed.), Dictionaries, Lexicography and Language Learning. Alemania: Janus Book, 61-68.

Castillo Carballo, M. ${ }^{a}$ A. (1998). "El término 'colocación' en la lingüística actual”, en Lingüistica Española Actual, 20, 1: 41-54.

Corpas Arellano, M. D. (2000). "La mujer y las estrategias de aprendizaje en la adquisición de la lengua inglesa", en Revista Nebrija de Lingüistica Aplicada, 4, disponible en: http://www. nebrija.com/revista-linguistica/la-mujer-y-las-estrategias-de-aprendizajeen-la-adquisicion-dela-lengua-ingles, consultado el 3 de octubre de 2014.

Corpas Pastor, G. (1996). Manual de Fraseología española. Madrid: Gredos.

Corpas Pastor, G. (2001). "Apuntes para el estudio de la colocación”, en LEA, 23 (1): 41-56.

Coseriu, E. (1977). Principios de semántica estructural. Madrid: Gredos.

Ellis, N. C., Simpson-vlach, R., y Maynard, C. (2008) "Formulaic language in native and second language speakers: Psycolonguistics, corpus linguistics and TESOL", en TESOL Quarterly 42, 3: 375-396.

Fernández Lázaro, G. (2014). "Enseñanza y aprendizaje de las colocaciones en el nivel inicial A1-A2", en MarcoELE, 19: 1-16, disponible en: http://marcoele.com/colocaciones-en-nivelinicial/, consultado el 3 de octubre de 2014.

Firth, J. R. (1957). Papers in Linguistics 1934-1951. Londres: Oxford University Press.

Handl, S. (2008). "Essential collocations for learners of English: The role of collocational direction and weight", en F. Meunier y S. Grandger (eds.) Phraseoloy in Foreign Language Learning and Teaching. London: John Benjamins, 43-66.

Higueras García, M. (2006). Las colocaciones y su enseñanza en la clase de ELE. Madrid: Arco/Libros. Hill, J. (2000). "Revising priorities: from grammatical failure to collocational success" en M. Lewis (Ed.). Teaching collocation: Further development in the lexical approach. Oxford: Oxford University Press, 47-69.

Instituto Cervantes (2006). Plan curricular del Instituto Cervantes. Niveles de referencia para el español, Madrid: Biblioteca Nueva, disponible en: http://cvc.cervantes.es/ensenanza/ biblioteca_ele/plan_curricular/, consultado el 25 de septiembre de 2014.

Izquierdo Gil, M. C. (2004). La selección del léxico en la enseñanza del español como lengua extranjera. Su aplicación en el nivel elemental en estudiantes francófonos, Valencia: Servei de Publicacions de la Universidad de Valencia., disponible en: http://www.tdx.cesca.es/ TDX-0425105-135323, consultado el 6 de octubre de 2014. 
Jiménez Catalán, R. M. (2003). "Sex differences in L2 vocabulary learning strategies", en International Journal of Applied Linguistics, 13, 1: 54-77.

Koike, K. (2001). Colocaciones léxicas en el español actual, estudio formal y léxico semántico. Alcalá de Henares: Universidad de Alcalá-Takushoku University.

Krashen, S. (1987). Principles and Practice in Second Language Acquisition. Oxford: Pergamon.

Laufer, B. y Walkman, T. (2011). "Verb-Noun Collocations in Second Language Writing: A Corpus Análisis of Learner'English", en Language Learning, 61, 2: 647-672.

Lewis, M. (ed.) (2000). Teaching collocation. Further developments in the Lexical Approach. London: Commertial Colour Press.

Ministerio de Educación, Cultura y Deporte (2002). Marco común europeo de referencia para las lenguas: aprendizaje, enseñanza y evaluación, disponible en: http://cvc.cervantes.es/ ensenanza/biblioteca_ele/marco/, consultado el 25 de septiembre de 2014.

Nation, I. S. P. (2001). Learning Vocabulary in Another Language. Cambridge: Cambridge University Press.

Nesselhauf, N. (2003). "The Use of Collocations by Advanced Learners of English and Some Implications for Teaching", en Applied Linguistics, 24, 2: 223-242.

Pavlenko, A. (1999). "New approaches to concepts in bilingual memory", en Bilingualism: Language and Cognition, 2: 209-230.

Pavlenko, A. (2001). "How am I to become a woman in an American vein?: Transformations of gender performance in second language learning", en A. Pavlenko, A. Blackledge, I. Piller y M. Teutsch-Dwyer (eds.), Multilingualism, second language learning, and gender. Berlin: Mouton De Gruyter, 133-174.

Pavlenko, A. (2004). "Gender and sexuality in foreign and second language education: Critical and feminist approaches" en B. Norton y K. Toohey (eds.), Critical pedagogies and language learning. Cambridge: Cambridge University Press, 53-71.

Pear S. y Barret B. S. (2013). "Rol of gender in spanish L2 reading", en Diálogo de la Lengua, $\mathrm{V}: 14-32$.

Pear, S. (2011). "El aprendizaje de vocabulario en español como L2 a través del uso de la tecnología. Un análisis de género", en Porta Linguarum 16: 179-192.

Rodríguez- Pérez. N. (2014). "Creencias y representaciones de los profesores de lenguas extranjeras sobre la influencia de los factores motivacionales y emocionales en los alumnos y en las alumnas", en Porta Linguarum 21: 183-197.

San Mateo Valdehíta, A. (2012). Aprendizaje de léxico en español como segunda lengua/lengua extranjera: investigación sobre la efectividad de tres tipos de actividades para aprender vocabulario (tesis doctoral inédita). Universidad Nacional de Educación a Distancia.

Sinclair, J. (1991). Corpus, Concordance, Collocation. Oxford: Oxford University Press.

Singleton, D. (1999). Exploring the second language mental lexicon. Cambridge: Cambridge University Press.

Travalia, C. (2006). "Las colocaciones implícitas", en Estudios de Lingüística de la Universidad de Alicante, 20: 317-332.

Webb, S. y Kagimoto, E. (2011). "Learning Collocations: Do the Number of Collocates, Position of the Node Word, and Synonymy Affect Learning?", en Applied Linguistics, 32, 2: 259-276.

Wei, L. (2003). "Activation of Lemmas in the Multilingual Mental Lexicon and Transfer in third Language Learning”, en J. Cenoz, B. Hufeisen y U. Jessner (eds.) The Multilingual Lexicon. Netherlands: Kluwer Academic Publishers, 57-70.

Wharton, G. (2000). "Language Learning Strategy Use of Bilingual Foreign Language Learners in Singapore" en Language Learning, 50, 2: 203-243.

Woolard, G. (2000). "Collocation-encouraging learner independence", en M. Lewis (ed.). Teaching collocation: Further development in the lexical approach. Oxford: Oxford University Press, 28-46. 